\title{
Schwannoma of Superficial Peroneal Nene Presenting as Leg Pain
}

\author{
Manik Mahajan, Rajesh Sharma, Poonam Sharma', Anchal Gupta. \\ From the Department of Radio-diagnosis and Imaging, ASCOMS Hospital, \\ University of Jammu and Department of Pathology', GMC Hospital, \\ University of Jammu, Jammu (J\&K), India
}

Abstract:

Schwannomas (or neurilemmomas) are lobulated, encapsulated tumours that arise from the neurilemmal cells in nerve sheaths. Schwannomas of the superficial peroneal nerves are a rare occurrence and a very few cases have been reported so far. Magnetic resonance imaging offers a precise diagnosis of the tumour. Herein, we report the Computed Tomography and Magnetic Resonance Imaging findings of superficial peroneal nerve schwannoma below the fibular neck in a middle aged female patient complaining of pain and tenderness in the lateral aspect of left leg.

Key words: Schwannoma, Magnetic Resonance Imaging, Peroneal nerve

\section{Introduction}

Schwannomas or neurilemmomas are lobulated, encapsulated tumours that arise from the neurilemmal cells in nerve sheaths [1]. Schwannomas arise from the myelin sheath of nerves and are the most common solitary nerve tumour of the body [2]. They usually arise in peripheral nerves or nerve roots and are relatively slow growing tumours. They may occur singly or in multiple, and have been found in a number of locations. They are normally benign; less than one percent become malignant, degenerating into a form of cancer known as neurofibrosarcoma. Schwannomas usually are solitary and occur at random; however, in some patients they can be related to a hereditary disorder. Schwannomas can be found in various parts of the body with the most common site being the head. In the lower extremity they are most commonly found in the deep tissues of the foot [3]. Schwannoma of the superficial peroneal nerve is very rare, with only four cases reported till 2006, according to Medline database [4-6].

Here we describe the Computed Tomography (CT) and Magnetic Resonance Imaging (MRI) findings in a case of schwannoma of superficial peroneal nerve below the level of fibular neck in a middle aged female patient presenting with pain, tenderness and paraesthesias in the lateral aspect of left leg.

\section{Case Report}

A 52 years old female presented to an orthopaedician with chief complaints of pain and paraesthesias in the lateral aspect of upper one third of left leg. The pain was moderate in intensity, burning in character and off and on in nature. There was no diurnal variation or radiation of pain. On examination, there was moderate tenderness in the same region. There was reduced sensation along the lateral aspect of the left leg. Computed Tomography (CT) and Magnetic Resonance Imaging (MRI) of the leg were advised for further evaluation. CT revealed a well defined round to oval soft tissue density lesion in the peroneal compartment on the lateral 
aspect of left leg below the fibular neck [Fig. 1]. MRI revealed a round to oval lesion measuring $9 \times 8 \mathrm{~mm}$ below the fibular neck in the peroneal compartment indenting the peroneus brevis muscle, exhibiting intermediate signal intensity on T2 weighted images [Fig. 2] and hyperintense signal on FSEIR image [Fig.3]. Later on patient was taken up for surgery. Excision of the lesion was performed and subjected to histopathological examination. Histology report described the lesion as a benign schwannoma with no evidence of malignant change [Fig. 4].
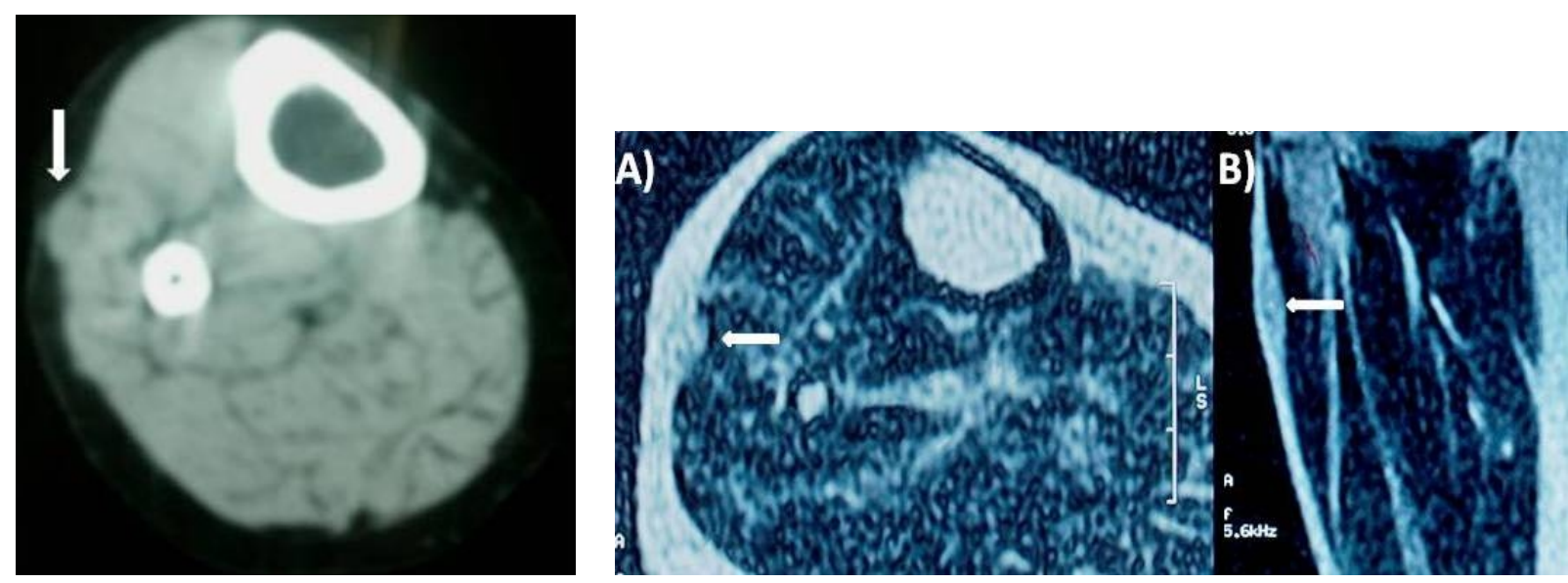

Fig. 1: Axial CT scan below the level of fibular neck showing a well defined round to oval soft tissue density lesion in the peroneal compartment on the lateral aspect of left leg (white arrow).

Fig. 2: Axial and Coronal T2 weighted MRI images showing a round to oval lesion intermediate signal intensity lesion below the fibular neck in the peroneal compartment indenting the peroneus brevis muscle (white arrows).
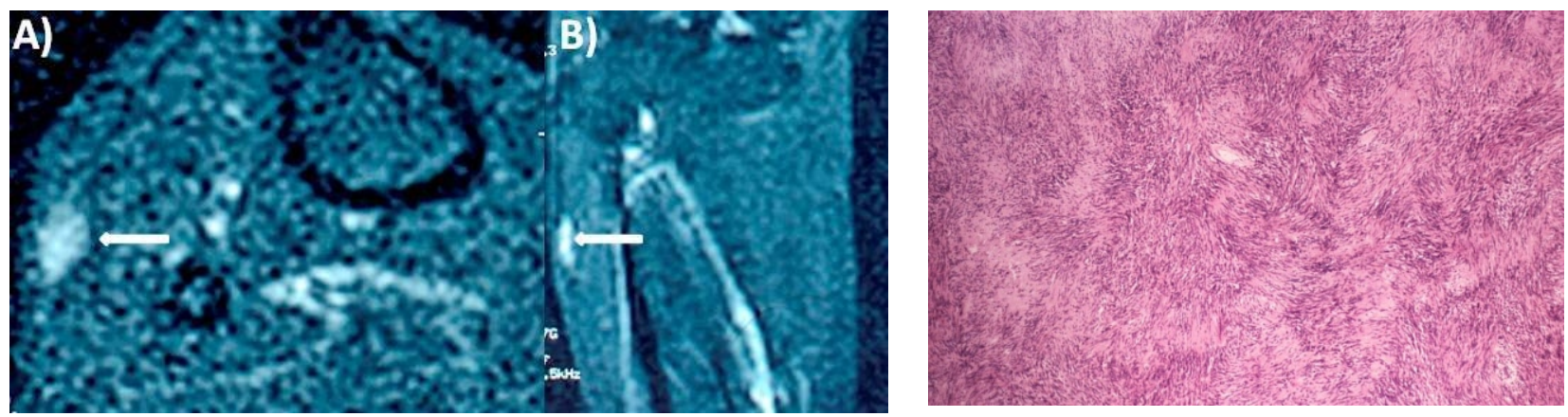

Fig. 3: Axial and Coronal FSEIR images showing a hyperintense signal intensity lesion indenting the peroneus brevis muscle in the peroneal compartment.

Fig. 4: Histological Slide (haematoxylin-eosin, low power magnification) showing areas of moderate to high cellularity and scant stromal matrix comprising of elongated cells with cytoplasmic processes arranged in fascicles along with less densely cellular areas consisting of loose meshwork of cells confirming the diagnosis of schwannoma. 


\section{Discussion}

Schwannomas or neurilemmomas are lobulated, encapsulated tumours that arise from the neurilemmal cells in nerve sheaths [1]. Schwannomas are derived from schwann cells of the neuroectoderm. Their function is to form the myelin sheath of nerves in the peripheral nervous system, which insulates the nerve and facilitates the transmission of an impulse. Also categorized with a neurinoma, neurilemmoma, or neurofibroma, the schwannoma is a benign encapsulated slow growing tumour [7-8]. Unlike neurofibromas, schwannomas do not traverse through the nerve but remain in the sheath lying on top of the nerve. They have a low risk of metastasizing. Schwannomas were found to have some transmission types that were autosomal dominant [8]. Schwannomas are most common in patients in the second through the fifth decades of life and have no gender or racial predilection [7].

Schwannomas can present with no symptoms, mild symptoms or severe symptoms mostly affecting the nerves. Most lesions are solitary and present as a slowly growing painless soft-tissue mass. Symptoms are unusual, unless the mass has become large enough to compress the adjacent nerve. Infrequently, these tumours can be associated with Neurofibromatosis 1, and in such cases, they are invariably plexiform or multiple lesions. The first case of a solitary schwannoma was discussed by Liebau, who stated that schwannomas should be looked for in all cases where patients present with pain, paresthesia of leg and foot, especially if all other injury has been excluded [9].

CT appearance of schwannomas has been described as a well circumscribed, homogeneous mass of soft tissue density. MRI is especially useful in identifying the exact location and size of the tumour. Schwannomas have isointense signal relative to skeletal muscle on $\mathrm{T} 1$-weighted images and increased slightly heterogeneous signal intensity on T2-weighted images [ $10-11$ ] . On FSEIR images, they have bright signal intensity. Degeneration and cystic cavitations are common in schwannomas. "Ancient" schwannomas refer to long-standing lesions with advanced degeneration exhibiting calcification, hyalinization, and cystic cavitation, findings that can be identified on imaging [12]. Pathologically, schwannomas are fusiform masses that are eccentrically located in relation to the involved nerve and are contained within a capsule, the epineurium. They are composed primarily of Schwann's cells. Surgical excision can usually spare the parent nerve because the schwannoma is generally separable from the underlying nerve fibres.

In conclusion, superficial peroneal nerve schwannomas are a very rare solitary nerve sheath tumours. They should always be considered as a differential diagnosis when tarsal tunnel syndrome, neuromas, nerve entrapment or radiculopathy is suspected. Schwannomas found in the proximal aspect of the lower extremity can also cause distal symptoms or injury, so this must also be considered, especially if the previous differentials have been ruled out. Early diagnosis can prevent permanent nerve damage, soft tissue or bony deformity. Thus in the differential diagnosis of non traumatic and non arthritic pain of lower leg and foot, benign tumours especially schwannomas of the peroneal nerves should always be considered.

\section{Consent}

The patient has given his or her informed consent for the case report to be published 


\section{References}

1. Russell RCG, Williams NS and Bulstrode CJK. Bailey and Love's Short Practice of Surgery. 23rd Ed, London: Arnold; 2000.

2. Stout AP: Tumors of the peripheral nervous system. In: Atlas of tumor pathology. Section 2, Fasicl 6 . Washington, D.C., Armed Forces Institute of Pathology, 1949.

3. Takada E, Ozaki T, Kunisada T, Harada Y, Inove H: Giant schwannoma of the back. Arch Orthop Trauma Surg. 2000;1 20: 467-469.

4. Houshian S, Freund KG. Gigantic benign schwannoma in the lateral peroneal nerve. Am J Knee Surg 1999; 12: $41-42$.

5. Laurencin CT, Bain M, Yue JJ, Glick H. Schwannoma of the superficial peroneal nerve presenting as web space pain. J Foot Ankle Surg 1995; 34: 532-533.

6. Thomas GP, Baco AM, Rosenfeld P. Schwannoma of the superficial peroneal nerve. Internet J Orthop Surg. 2006; 3(2): 12.

7. Joyce M, Laing AJ, Mullet H, Mofidi A, Tansey D, Connolly CE, McCabe JP. Multiple schwannomas of the posterior tibial. Nerve Foot Ankle Surgery2002; 8:1 $01-103$.

8. Liebau C, Baltzer AW, Schneppenheim M, Braunstein S, Koch H, Merk H. Isolated peripheral neurilemoma attached to the tendon of the flexor digitorum longus muscle. Arch Orthop Trauma Surg 2003;1 23: 98-101.

9. White NB: Neurilemomas of the extremities J Bone Joint Surg. 1967;49A:1605-1610.

10. Hems TE, Burge PD, Wilson DJ. The role of magnetic resonance imaging in the management of peripheral nerve tumours. J Hand Surg $[\mathrm{Br}] 1997 ; 22: 57-60$.

11. Stull MA, Moser RP, Kransdorf MJ, Bogumill GP, Nelson MC. Magnetic resonance appearance of peripheral nerve sheath tumors. Skeletal Radiol. $1991 ; 20: 9-14$.

12. Schultz E, Sapan MR, McHeffey-Atkinson B, Naidich JB, Arlen M. Case report 872. "Ancient" schwannoma (degenerated neurilemmoma). Skeletal Radiol 1994;23:593-595. 